\title{
Internal dynamics and particle acceleration in Tycho's SNR
}

\author{
H. J. Völk ${ }^{1}$, E. G. Berezhko², and L. T. Ksenofontov ${ }^{2}$
}

\author{
1 Max-Planck-Institut für Kernphysik, PO Box 103980, 69029 Heidelberg, Germany \\ e-mail: Heinrich.Voelk@mpi-hd.mpg.de \\ 2 Yu. G. Shafer Institute of Cosmophysical Research and Aeronomy, 31 Lenin Ave., 677980 Yakutsk, Russia \\ e-mail: berezhko@ikfia.ysn.ru
}

Received 28 December 2007 / Accepted 3 March 2008

\begin{abstract}
Aims. The consequences of a newly suggested value for the SN explosion energy $E_{\mathrm{sn}}=1.2 \times 10^{51}$ erg are explored for the case of Tycho's supernova remnant (SNR).

Methods. A nonlinear kinetic theory of cosmic ray (CR) acceleration in supernova remnants (SNRs) is employed to investigate the properties of Tycho's SNR and their correspondence to the existing experimental data.

Results. It is demonstrated that the high mean ratio between the radii of the contact discontinuity and the forward shock is consistent with the very effective acceleration of nuclear energetic particles at the forward shock. It is also argued that consistency of the value $E_{\mathrm{sn}}=1.2 \times 10^{51} \mathrm{erg}$ with the gas dynamics, acceleration theory, and the existing $\gamma$-ray measurements requires the source distance $d$ to be greater than $3.3 \mathrm{kpc}$. The corresponding ambient gas number density is lower than $0.4 \mathrm{~cm}^{-3}$. Since the expected $\gamma$-ray flux strongly depends on the source distance, $F_{\gamma} \propto d^{-7}$, a future experimental determination of the actual $\gamma$-ray flux from Tycho's SNR will make it possible to determine the values of the source distance $d$ and of the mean ambient gas density. A simple inverse Compton model without a dominant population of nuclear CRs is not compatible with the present upper limit for the $\gamma$-ray emission for any reasonable ambient interstellar $B$-field.

Conclusions. Given the consistency between acceleration theory and overall, as well as internal, gas dynamics, a future $\gamma$-ray detection would make the case for nuclear particle acceleration in Tycho's SNR incontrovertible in our view.
\end{abstract}

Key words. ISM: cosmic rays - acceleration of particles - shock waves - stars: supernovae: individual: Tycho's SNR radiation mechanisms: non-thermal - gamma-rays: theory

\section{Introduction}

Nonlinear kinetic theory of diffusive CR acceleration in SNRs (Berezhko et al. 1996; Berezhko \& Völk 1997) couples the gas dynamics of the explosion with the particle acceleration. The present form of the solution assumes spherical symmetry. In this approximation it is possible to predict the evolution of gas density, pressure, and mass velocity, as well as the radii of the forward shock and the contact discontinuity, together with the energy spectrum and the spatial distribution of CR nuclei and electrons, including the properties of their non-thermal radiation. Applied to individual young SNRs (see Völk 2004; Berezhko 2005,2008 , for reviews) this theory has successfully explained many observed SNR properties. Making use of the observed synchrotron emission spectrum from radio to X-ray frequencies it permits the derivation of the injection rate of nuclear particles, essentially that of protons, into the acceleration process. It has also allowed the determination of the degree of magnetic field amplification, a process advocated earlier from plasma simulations (Lucek \& Bell 2000; Bell \& Lucek 2001). The initial examples of such a systematic analysis concerned SN 1006 (Berezhko et al. 2002) and Tycho's SNR (Völk et al. 2002) ${ }^{1}$. It was specifically predicted that magnetic field amplification leads to the concentration of the highest-energy electrons and their corresponding synchrotron and IC $\gamma$-ray emission in a very thin shell just

${ }^{1}$ First indications for high magnetic field strengths $B=10^{2}-10^{3} \mu \mathrm{G}$ had been obtained much earlier for Tycho's SNR by Reynolds \& Ellison (1992) from an analysis of the radio spectrum alone. behind the forward shock (see Fig. 7 of Berezhko et al. 2002). This concentration, which is a result of synchrotron cooling, is observationally broadened due to a projection effect (Berezhko et al. 2003) $)^{2}$.

As indicated above, the theory has been used in some detail to investigate Tycho's SNR (G120.1+1.4) as the remnant of a type Ia SN in a (roughly) uniform interstellar medium (ISM), in order to compare the results with existing data. A stellar ejecta mass $M_{\mathrm{ej}}=1.4 M_{\odot}$, distance $d=2.3 \mathrm{kpc}$, and ISM number density $N_{\mathrm{H}}=0.5 \mathrm{H}$-atoms cm${ }^{-3}$ (Völk et al. 2002, 2005b) were used. For these parameters a total hydrodynamical explosion energy $E_{\mathrm{sn}}=0.27 \times 10^{51}$ erg was originally derived to fit the observed size $R_{\mathrm{s}}$ and expansion speed $V_{\mathrm{s}}$.

The steep and concave radio synchrotron spectrum shows that the forward shock is characteristically modified by the pressure of the accelerated nuclear particle component, with the weaker subshock essentially accelerating the radio electrons, while higher-energy electrons increasingly "see" the overall shock transition (Reynolds \& Ellison 1992; Berezhko et al. 2002).

\footnotetext{
${ }^{2}$ Such filamentary structures have been observed in hard X-rays (Vink \& Laming 2003; Long et al. 2003; Bamba et al. 2003) and are nowadays used as a second independent method to infer the magnitude of the amplified field. As it should be, both methods lead to consistent results within the errors, whenever both data sets exist (Vink \& Laming 2003; Berezhko \& Völk 2004; Völk et al. 2005b; Ballet 2006; Parizot et al. 2006).
} 
Since the hardening of the particle momentum spectrum begins at momenta $p \approx m_{\mathrm{p}} c$, the frequency range, where the corresponding hardening of the spatially integrated synchrotron spectrum occurs, brackets the effective mean magnetic field strength $B_{\mathrm{d}}$ inside the SNR; here $m_{\mathrm{p}}$ denotes the proton mass. The magnetic field strength is in addition constrained by the requirement that the calculated electron spectrum leads to a smooth synchrotron cutoff whose frequency agrees with the X-ray observations. From such a comparison of the calculated and observed overall synchrotron spectra the field strength resulted as $B_{\mathrm{d}} \approx 240 \mu \mathrm{G}$. Assuming the mean magnetic field strength in the circumstellar medium of Tycho's SN to be equal to a typical ISM-value of $5 \mu \mathrm{G}$, and using an overall shock compression ratio $\sigma<6$ (see below), pure MHD-compression would give a much smaller interior field strength $B_{\mathrm{d}}<30 \mu \mathrm{G}^{3}$. A later re-analysis, including now independent Chandra observations of thin X-ray filaments as indicators of the outer shock, gave $B_{\mathrm{d}} \approx 300 \pm 60 \mu \mathrm{G}$ (Völk et al. 2005b,a). Overall, together with the approximation for the scattering properties of the magnetic field fluctuations - summarized in the next section - good consistency of the predictions of the nonlinear theory with the existing observational data was achieved.

Recently the radius $R_{\mathrm{c}}$ of the contact discontinuity (CD), separating the shocked ISM and the ejecta material, has been investigated by Warren et al. (2005), using Chandra X-ray observations. The ratio $R_{\mathrm{c}} / R_{\mathrm{S}}$ of $R_{\mathrm{c}}$ and the radius $R_{\mathrm{S}}$ of the forward shock (blast wave) is a new physical variable characterizing Tycho's SNR. It connects the internal dynamics with the particle acceleration process. The large mean value $R_{\mathrm{c}} / R_{\mathrm{S}}=0.93$ found for this ratio was interpreted as evidence for efficient CR acceleration which makes the medium between those two discontinuities considerably more compressible. Without accelerated particles, and in a spherically symmetric calculation, the value of the ratio is significantly smaller, $R_{\mathrm{c}} / R_{\mathrm{S}}=0.77$ (Wang \& Chevalier 2001). We have demonstrated (Völk et al. 2007, 2008) that the observed ratio $R_{\mathrm{c}} / R_{\mathrm{S}}=0.93$ agrees quite well with the results of Völk et al. (2002).

Somewhat later Badenes et al. (2006) published a detailed comparison between the high-quality X-ray observations from XMM-Newton and Chandra of the ejecta emission and current models for Type Ia explosions. They found that the fundamental properties of the X-ray emission in Tycho are well reproduced by a one-dimensional delayed detonation model with a kinetic energy $E_{\mathrm{sn}}=1.2 \times 10^{51} \mathrm{erg}$. Such a value is compatible with well-known results for deflagration models (Nomoto et al. 1984) as well. In any case, this explosion energy is rather in the middle of the typical range of type Ia SN explosion energies that vary by a factor of about two (e.g. Gamezo et al. 2004; Blinnikov et al. 2006). In our initial paper (Völk et al. 2007) we had indicated that also such a higher kinetic energy of the explosion is consistent with the SNR dynamics.

Here we shall a priori adopt the value $E_{\mathrm{sn}}=1.2 \times 10^{51} \mathrm{erg}$ and perform the calculation of all relevant physical properties of Tycho's SNR connected with nonthermal energy production and emission in order (i) to find out how well the existing SNR data - especially the ratio $R_{\mathrm{c}} / R_{\mathrm{S}}$ - are consistent with such an explosion energy (ii) to investigate the expected ratio of leptonic to hadronic $\gamma$-ray emission, and (iii) to possibly determine the

3 Since injection of nuclear particles occurs only at quasi-parallel shocks (Völk et al. 2003), a pure MHD-compression of a $5 \mu \mathrm{G}$ upstream field would lead to even considerably lower interior field strengths $\sim 10 \mu \mathrm{G}$ in an unmodified shock with a compression ratio of 4 . This property will be used in Sect. 3.1. allowed range for the source distance. In Sect. 2 we shall outline our approximation for the scattering properties of the magnetic field fluctuations, whereas Sect. 3 contains the results and the discussion.

\section{Approximation for the scattering properties of the system}

Recently Bell (2004) found that a non-resonant streaming instability will occur within the precursor of a strong, accelerating shock. The diffusive streaming of accelerated CRs is expected to be so strong in this region that a purely growing MHD mode appears with a growth rate that is, at least at the beginning of the precursor, larger than the growth rate of the well-known resonant Alfvénic mode. It is expected that due to this non-resonant instability the external magnetic field $B_{\text {ISM }}$ is amplified within the entire precursor structure, since the main effect is produced by the most energetic CRs which populate the whole precursor diffusively during their acceleration. The Alfvén wave excitation in the shock precursor (Bell 1978; Blandford \& Ostriker 1978; McKenzie \& Völk 1982) corresponds to an additional unstable mode that in particular leads to a high level of resonantly scattering waves. This mode has been argued to dominate the magnetic field energy deep in the precursor, after saturation of the nonresonant instability (Pelletier et al. 2006). Therefore the overall field amplification will be the result of both instabilities operating in the precursor.

The saturation value $B_{\mathrm{nr}}$ of the non-resonantly amplified magnetic field is given by (Bell 2004; Pelletier et al. 2006)

$\frac{B_{\mathrm{nr}}^{2}}{8 \pi} \sim \frac{3 V_{\mathrm{s}} P_{\mathrm{c}}}{2 \Phi c} \approx 1.5 \times 10^{-3} P_{\mathrm{c}}\left(\frac{V_{\mathrm{s}}}{3000 \mathrm{~km} \mathrm{~s}^{-1}}\right)$,

where $P_{\mathrm{c}}$ is the resulting CR pressure, $\Phi=\log \left(\epsilon_{\max } / m_{\mathrm{p}} c^{2}\right), \epsilon_{\max }$ is the maximal CR energy, $m_{\mathrm{p}}$ is the proton mass, and the value $\Phi=10$ was adopted in the above relation. This value of $B_{\mathrm{nr}}^{2} /(8 \pi)$ is by a factor $\approx 3.3$ smaller than the value

$B_{0}^{2} /(8 \pi) \approx 5 \times 10^{-3} P_{\mathrm{c}}$

empirically determined for several SNRs, where the upstream (amplified) magnetic field strength $B_{0}$ in the shock precursor, defined as $B_{0}=B_{\mathrm{d}} / \sigma$, has been derived both from the spatially integrated radio and $\mathrm{X}$-ray synchrotron spectrum, as well as from the thickness of the X-ray filaments which determine the downstream field strength $B_{\mathrm{d}}$ (Berezhko \& Völk 2006).

The subsequent growth of the resonant Alfvén instability towards the precursor takes place in a medium where the effective Alfvén velocity is given by $c_{\mathrm{A}}=B_{\mathrm{nr}} / \sqrt{4 \pi \rho}$ and $\rho$ is the mass density. Following Pelletier et al. (2006, see their Sect. 3.2.2.) one obtains at the subshock

$\frac{B_{\mathrm{res}}^{2}}{B_{0}^{2}} \approx 7\left(\frac{V_{\mathrm{s}}}{3000 \mathrm{~km} \mathrm{~s}^{-1}}\right)^{1 / 2}$

This expression shows that the existing mechanisms are able to amplify the magnetic field within the precursor to a strength, which considerably exceeds the required value (2) (Berezhko 2008). In fact, it would be more appropriate to use in Eq. (3) instead of $B_{0}$ the value $B_{1}=\left(\sigma / \sigma_{\mathrm{s}}\right) B_{0}$, which in our model is the field strength just ahead of the subshock, where $\sigma_{\mathrm{s}}$ is the subshock compression ratio. This reduces the numerical factor 7 on the r.h.s. of Eq. (3) to about 4 for an object like Tycho's SNR, which still substantially exceeds the required value. However, 
this contribution $B_{\text {res }}^{2} /(8 \pi)$ of the resonant instability to the magnetic field energy density disregards any nonlinear wave dissipation. Indeed, within the framework of weak plasma turbulence theory, the resonant instability is growing freely up to the subshock, where the growth rate goes to zero abruptly.

However, the MHD simulations by Bell (2004), recently confirmed with higher numerical resolution by Zirakashvili et al. (2008), show strong dissipation of the excited non-resonant instability into the thermal plasma by induced gas compressions which dissipate in shocks. At the same time the field strength is expected to reach a value $B_{\mathrm{nr}} \gg B_{\mathrm{ISM}}$, discussed above, at which the instability saturates (for a different view, see Niemiec \& Pohl 2007). It is true that these simulations do not directly describe the situation in the nonuniform shock precursor with its mixture of non-resonant and resonant fluctuations, where closer to the subshock more and more resonant particles appear. Nevertheless the shock dissipation of transverse magnetic turbulence into the thermal gas is likely to be a generic feature for the mixture of non-resonant and resonant instability modes, and should in particular also effectively dissipate the developing resonant waves.

This theoretical expectation of strong nonlinear wave dissipation is consistent with Eq. (2) which shows that the uninhibited growth of the resonant instability leads to a substantial overestimate for the magnetic wave energy density at the subshock. It is therefore a reasonable approximation that at some level of magnetic field amplification beyond non-resonant saturation the nonlinear dissipation by shock formation balances the linear growth of the resonant instability, the remaining energy going into thermal energy of the precursor plasma.

The spectrum of CRs produced by a strong, modified shock is very hard so that the CRs with the highest energies make the largest contribution to the overall CR energy density. These most energetic CRs produce a field amplification on their own spatial scale which is the precursor size. Therefore CRs with lower energies already "see" the amplified field $B_{0}$ as a mean field. Beyond that the strongly excited resonant waves lead to strong particle scattering that approaches the Bohm limit, where the scattering mean free path locally equals the gyro radius in the amplified field $B$.

Since the process of magnetic field amplification is not included in our kinetic theory we postulate the existence of a far upstream amplified field $B_{0} \gg B_{\text {ISM }}$ that is determined from observations of the downstream magnetic field strength $B_{\mathrm{d}}$, and a simplified connection

$B=\left(\rho / \rho_{\mathrm{d}}\right) B_{\mathrm{d}}$

between the local field strength $B$ and the local density $\rho$. Together with the above equation for $B_{0}$, this defines the amplified magnetic field everywhere in the shock transition and is used in our models for the particle acceleration and gamma-ray emission (e.g. Berezhko 2008).

A value $B_{0}$ of the order of $10 B_{\text {ISM }}$ or even larger, as derived in the present paper (and for other sources: Völk et al. 2005b; Berezhko 2005, 2008), appears quite plausible for young objects, in which longer-term dissipation mechanisms (Ptuskin \& Zirakashvili 2003, 2005) have not yet had sufficient time to operate.

The problem is ultimately the scattering (or better, the scattering strength) of the highest-energy particles at the instantaneous cutoff. This is an intrinsically time-dependent process that is not yet resolved in particle acceleration theory even for unmodified shocks (e.g. Lagage \& Cesarsky 1983), let alone for nonlinearly modified shocks (Amato \& Blasi 2006).
This interpretation is different from that of Ptuskin \& Zirakashvili $(2003,2005)$ who were not yet aware of the nonresonant instability. It is also different from that of Amato \& Blasi (2006) and Vladimirov et al. (2006) who do not include the non-resonant instability and its field amplification effect in their arguments, and rather concentrate on the nonlinear effects of the resonant Alfvén wave instability in the spirit of Bell \& Lucek (2001). It is finally also different from the recent calculation of the acceleration properties of shocks by Zirakashvili \& Ptuskin (2008) who base their work entirely on the non-resonant instability and its amplification properties.

Our approximation for young objects consists then first of all in assuming that the magnetic field strength is amplified everywhere around the shock in the sense that all accelerating particles experience the amplified field in the region upstream of the subshock. The actual strength of $B_{0}$ is phenomenologically determined by iteratively fitting the theoretically calculated electron spectrum to the observed synchrotron spectrum, and/or by the analysis of observed X-ray filaments (e.g. Völk et al. 2005b). The effective downstream field strength $B_{\mathrm{d}}=\sigma B_{0}$ is the result of the MHD-compression of this largely perpendicular upstream field. On account of the large amplitude $\left\langle\delta B^{2}\right\rangle \sim B^{2}$ of the resonantly scattering waves, the diffusion coefficient is taken as the Bohm limit in the local amplified field $B=\left(\rho / \rho_{d}\right) B_{\mathrm{d}}$. This is clearly a lower limit to the scattering mean free path (e.g. Marcowith et al. 2006), especially for the highest-energy particles whose maximum energy is almost certainly overestimated in this way. However the Bohm limit seems to be roughly consistent with the observations (Parizot et al. 2006).

Secondly, we assume the dissipation rate of magnetic field energy into the thermal gas to be equal to the linear growth rate $c_{\mathrm{A}} \nabla P_{\mathrm{c}}$ of the resonant Alfvénic wave field, with $c_{\mathrm{A}}=$ $B / \sqrt{4 \pi \rho}$ (see also Berezhko 2008). This does not directly include the dissipative limitation of the growth of the non-resonantly unstable modes demonstrated by the MHD simulations and to that extent still underestimates the overall gas heating. The contribution of the non-resonant modes to the gas heating is only taken into account indirectly through the enhancement of the Alfvén velocity in the dissipation term.

For Tycho's SNR, as for all other cases studied, this gas heating lowers the overall compression ratio to values of the order of 5 to 7 which seem very plausible numbers in the light of the properties of the synchrotron emission.

It is clear from these arguments that the complexity of the magnetic field, even in the asymptotic case of strong shocks, can at present only be partially resolved from plasma theory alone. The observed synchrotron emission properties of SNRs however determine our key parameter $B_{0}$ which we use to give an approximate description as outlined above. This does not yet address the questions of the time dependence of this quantity (due to the higher shock velocity in the past) or of the latetime escape of high-energy particles. In this paper we shall assume that $B_{0}$ is a constant and fixed by the measurements at the present time. We note however that even a simplified picture with time-independent upstream field $B_{0}$ contains such an important element as escaping CRs (Berezhko \& Krymsky 1988; Berezhko et al. 1996) which are the highest-energy CRs whose outward diffusion is faster than the SNR shock expansion. In reality one has to expect this loss process to be even much faster due to two factors. First of all, a decreasing $B_{0}(t)$ due to the shock deceleration makes high-energy CR diffusion progressively faster. Secondly, these escaping CR particles that exist far upstream from the SN shock produce less efficiently magnetic field perturbations and therefore their diffusion coefficient 
Table 1. Models parameters (Tycho SNR).

\begin{tabular}{ccccccc}
\hline \hline$d, \mathrm{kpc}$ & $N_{\mathrm{H}}, \mathrm{cm}^{-3}$ & $\sigma$ & $B_{\mathrm{d}}, \mu \mathrm{G}$ & $B_{\mathrm{d}}^{\prime}, \mu \mathrm{G}$ & $K_{\mathrm{ep}}, 10^{-4}$ & $F_{\gamma}^{\pi} / F_{\gamma}^{\mathrm{IC}}$ \\
\hline 3.1 & 0.5 & 5.25 & 420 & 360 & 4.2 & 40 \\
3.3 & 0.4 & 5.15 & 412 & 350 & 5.1 & 27 \\
4.0 & 0.2 & 4.85 & 388 & 320 & 9.5 & 7.7 \\
4.5 & 0.1 & 4.6 & 368 & 309 & 15. & 2.4 \\
\hline
\end{tabular}

becomes progressively closer to the average diffusion coefficient in the ISM.

\section{Results and discussion}

The distance determinations for Tycho's SNR have varied in the past. De Vaucouleurs (1985) gave a most likely range as $d=3.2 \pm 0.3 \mathrm{kpc}$. Albinson et al. (1986) found $2.2_{-0.6}^{+1.5} \mathrm{kpc}$, whereas Schwarz et al. (1995) argued that $d$ could be as large as $4.5 \mathrm{kpc}$. Using $\mathrm{H}_{\alpha}$ measurements and assuming a strong gas shock with a compression ratio of $\sigma=4$ Kirshner et al. (1987) derived distances of 2.0-2.8 kpc, and Smith et al. (1991) extended this to $1.5-3.1 \mathrm{kpc}$. Very recently Ruiz-Lapuente (2004) estimated $d=2.83 \pm 0.79 \mathrm{kpc}$ by comparing Tycho's SNR to SN 1006, and $d=2.85 \pm 0.4 \mathrm{kpc}$ by using the peak luminositydecline correlation for type Ia Supernova explosions. With the exception of the upper limit of Schwarz et al. (1995), all these estimates lie below about $3.6 \mathrm{kpc}$. In such a situation we explore here the range of the distances $d=3.1-4.5 \mathrm{kpc}$. As the lowest value $d=3.1 \mathrm{kpc}$ is taken because distances $d \leq 3.1 \mathrm{kpc}$ are inconsistent with existing $\mathrm{TeV} \gamma$-ray measurements, as shown below.

For any given value $d$ we find the density $N_{\mathrm{H}}$ of the ambient ISM from a fit to the observed SNR size and expansion speed. Due to the relatively small uncertainties of the measurements of angular radius $R_{\mathrm{S}} / d$ and expansion speed $V_{\mathrm{s}} / d$, there exists an almost unique value of $N_{\mathrm{H}}$ for every given distance $d$, given the explosion energy $E_{\mathrm{sn}}$.

Figure 1 shows the calculations of shock-related hydrodynamic quantities for four different source distances in the range $3.1 \leq d \leq 4.5 \mathrm{kpc}$, together with the azimuthally averaged experimental data from radio observations (Tan \& Gull 1985). The corresponding ambient gas densities, which result from a fit to the observed angular SNR size and expansion speed, are listed in Table 1 together with the values of other relevant parameters at the current epoch.

The system is just in the transition from free expansion into the Sedov phase. Therefore, although inevitably rough, an analytical explanation of the resulting relation between $N_{\mathrm{H}}$ and $d$ is the following: since in the Sedov phase $R_{\mathrm{S}} \propto\left(E_{\mathrm{sn}} / N_{\mathrm{H}}\right)^{1 / 5}$, the density $N_{\mathrm{H}} \propto E_{\mathrm{sn}} / d^{5}$ strongly decreases with increasing distance $d$.

As in our previous studies (Völk et al. 2002, 2005b, 2007) we determine the values of the proton injection rate $\eta$, electron-toproton ratio $K_{\mathrm{ep}}$ and interior magnetic field value $B_{\mathrm{d}}$ from a fit to the observed synchrotron spectrum. The softness of the observed low-energy radio spectrum - relative to a test particle spectrum requires a proton injection rate $\eta=3 \times 10^{-4}$ in all cases for different distances. This implies a significant nonlinear modification of the shock at the current age of $t=435$ yrs (see Fig. 1b).

To fit the measured synchrotron flux a large magnetic field $B_{\mathrm{d}} \sim 400 \mu \mathrm{G}$ is required (see Table 1 ). Only such a high magnetic field strength can provide sufficiently large synchrotron losses to fit the observed X-ray flux. As Fig. 2 shows, one then can obtain a fit to the synchrotron radio and X-ray spectra which

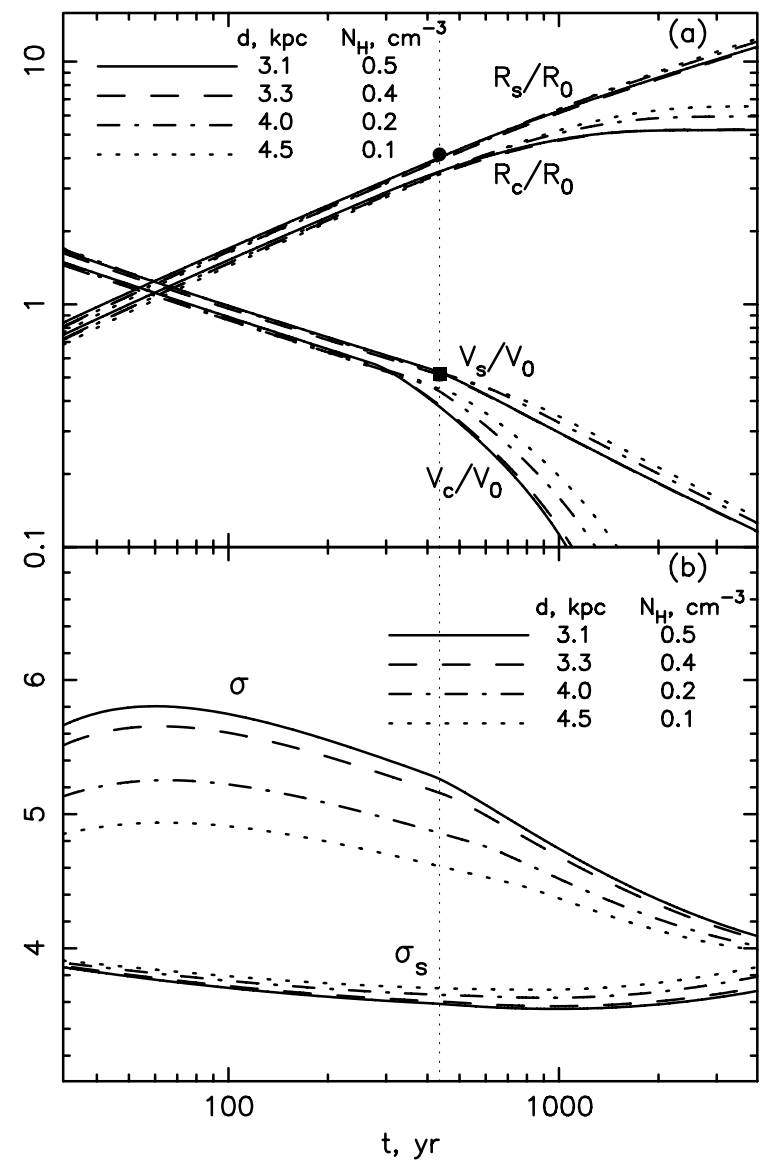

Fig. 1. a) Shock (contact discontinuity) radius $R_{\mathrm{S}}\left(R_{\mathrm{c}}\right)$ and shock (contact discontinuity) speed $V_{\mathrm{s}}\left(V_{\mathrm{c}}\right)$ in units of $R_{0}=(4 \mathrm{kpc} / d) \mathrm{pc}$ and $\left.V_{0}=10^{4}(4 \mathrm{kpc} / d) \mathrm{km} \mathrm{s}^{-1} ; \mathbf{b}\right)$ total shock $(\sigma)$ and subshock $\left(\sigma_{\mathrm{s}}\right)$ compression ratios for Tycho's SNR as functions of time, calculated for four different distances $d$. The dotted vertical line marks the current epoch, $t=435$ yrs. The observed mean size (filled circle) and speed (filled square) of the shock, as determined by radio measurements (Tan \& Gull 1985), are shown as well.

is as good as in our previous studies (Völk et al. 2002, 2005b), which assumed $E_{\mathrm{sn}}=0.27 \times 10^{51} \mathrm{erg}$ and in Völk et al. (2002) also a slight overestimate of the observed X-ray emission.

We now compare the theoretical ratio $R_{\mathrm{c}} / R_{\mathrm{S}}$ of the contact discontinuity radius (CD) and the blast wave radius with the observational estimate $R_{\mathrm{c}} / R_{\mathrm{S}}=0.93$ by Warren et al. (2005). In order to do this, one has to first make the two results comparable. It was argued in Völk et al. $(2007,2008)$ that if one starts from a spherically symmetric calculation of the $\mathrm{CD}$ radius as we do, one has to take into account that the actual CD is subject to the Rayleigh-Taylor instability, and thus a correction is needed to compare such a $1 \mathrm{D}$ calculation with observations of the $\mathrm{CD}$. Here we also correct our calculated CD radius by a factor 1.05 . It is clear that the increased compressibility of the shocked material can only be due to accelerated nuclear particles, because the pressure of the accelerated electrons is always negligibly small.

The comparison of the corrected values $R_{\mathrm{c}} / R_{\mathrm{S}}$ with the experimentally estimated value $R_{\mathrm{c}} / R_{\mathrm{S}}=0.93$ (in Fig. 3 we present that value with $2 \%$ uncertainties, according to Warren et al. 2005) shows quite good agreement in all the cases considered (see Fig. 3). According to the theoretical calculation the ratio $R_{\mathrm{c}} / R_{\mathrm{S}}$ is almost constant in the free expansion phase of the SNR evolution, whereas in the adiabatic phase the contact discontinuity quickly drops behind the forward shock and the ratio starts 


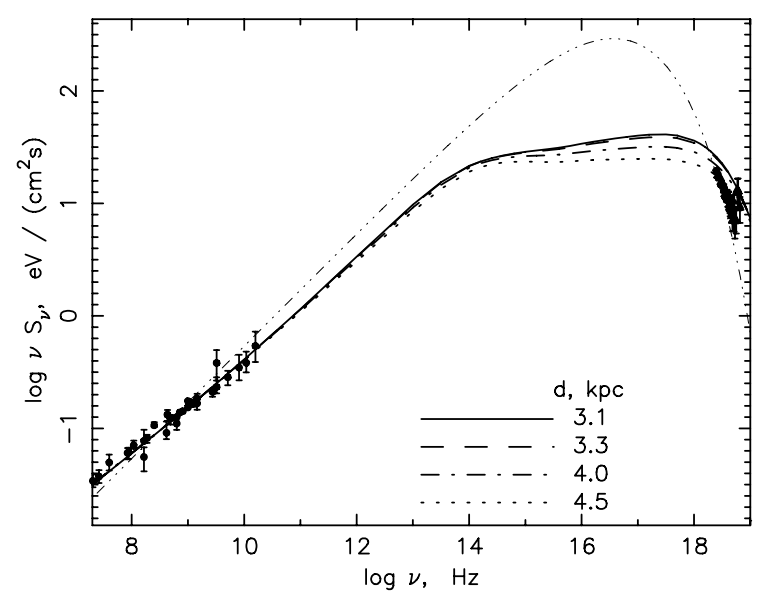

Fig. 2. Synchrotron emission flux as a function of frequency for the same four cases as in Fig. 1, at the current time. The thin dot-dot-dashed line represents the flux expected in the test particle limit for an interior magnetic field strength $B_{\mathrm{d}}=20 \mu \mathrm{G}$. The observed X-ray (Allen et al. 1999 ) and radio emission (Reynolds \& Ellison 1992) are shown as well.

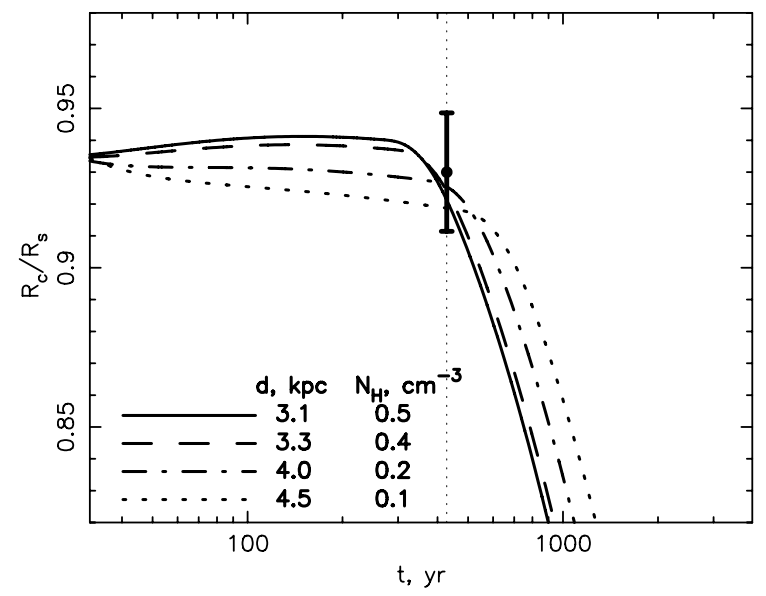

Fig. 3. The ratio $R_{\mathrm{c}} / R_{\mathrm{s}}$ of the contact discontinuity radius to the forward shock radius as a function of time, calculated for four different source distances, together with the observationally determined value of Warren et al. (2005).

to decrease. According to Figs. 1 and 3 Tycho's SNR is in transit to the adiabatic phase. In the case without $\mathrm{CR}$ acceleration our corrected value is $R_{\mathrm{c}} / R_{\mathrm{S}} \approx 0.89$. We note that this value is larger (and the effect of CRs correspondingly smaller) than that assumed by Warren et al. (2005), because these authors referred to SNR parameters according to which Tycho's SNR is closer to the adiabatic phase (Wang \& Chevalier 2001).

In order to find a constraint on the distance $d$ and the ISM density $N_{\mathrm{H}}$ from $\gamma$-ray observations, we compare in Fig. 4a the resulting $\gamma$-ray spectral energy distribution with the HEGRA and Whipple upper limits at TeV energies. The $\gamma$-ray fluxes, calculated in spherical symmetry, have all been reduced by a factor $f_{\text {re }}=0.2$, in order to account for the selective injection of protons which operates only over $\approx 20 \%$ of the SNR surface (Völk et al. 2003). It can be seen that all distances $d<3.3 \mathrm{kpc}$ are inconsistent with the HEGRA data ${ }^{4}$. We also note that at all distances considered the expected $\gamma$-ray flux is dominated by $\pi^{0}$-decay (hadronic) $\gamma$-rays (see Table 1 and Fig. 4b). However, the

\footnotetext{
${ }^{4}$ Given the considerably higher sensitivity of HEGRA, the SHALON results contradict the $\mathrm{H}-\mathrm{CT}$ result. Therefore we shall not discuss the claimed detection by SHALON.
}

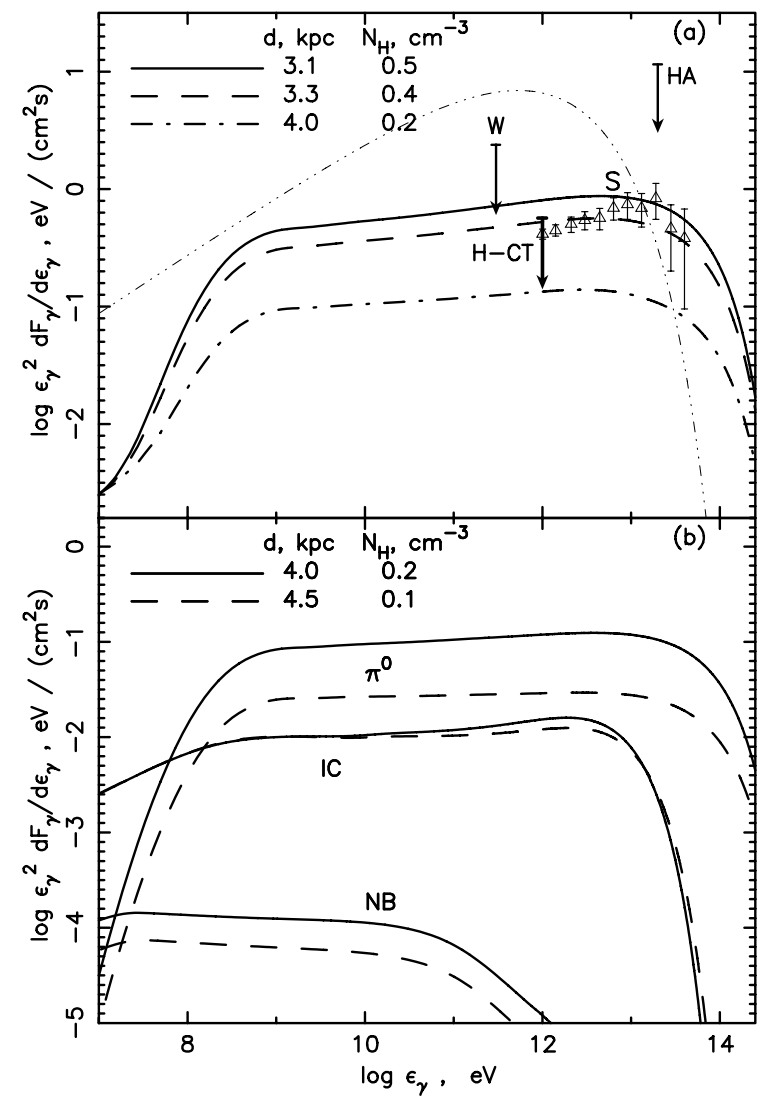

Fig. 4. Spectral energy distributions of the $\gamma$-ray emission from Tycho's SNR (total spectrum, which is the sum of all components, a), and separately $\pi^{0}$-decay, inverse Compton (IC) and non-thermal Bremstrahlung (NB) components, b)), as functions of $\gamma$-ray energy $\epsilon_{\gamma}$, calculated for different source distances, together with the upper limits measured by the HEGRA (H-CT; Aharonian et al. 2001) and Whipple (W; Buckley et al. 1998) Cherenkov telescopes, the HEGRA AIROBICC (HA; Prahl \& Prosch 1997) upper limit, and data presented by SHALON (S; Sinitsyna et al. 2007). The thin dot-dot-dashed line in Fig. 4a represents the IC spectrum that is expected to be produced in the test particle case.

contribution of these hadronic $\gamma$-rays progressively decreases with the increase of the source distance: the hadronic $\gamma$-ray flux $F_{\gamma}^{\pi} \propto E_{\mathrm{c}} N_{\mathrm{H}} / d^{2}$ is proportional to the ISM number density $N_{\mathrm{H}}$ and to the total CR energy content $E_{\mathrm{c}}$ (e.g. Berezhko \& Völk 1997). In the Sedov phase $E_{\mathrm{c}}$ is an almost distance-independent fraction of $E_{\mathrm{sn}}$, and $N_{\mathrm{H}} \propto d^{-5}$. This gives then $F_{\gamma}^{\pi} \propto d^{-7}$, since $E_{\text {sn }}$ is fixed. Such a dependence is roughly consistent with the results of our calculations given in Fig. 4 .

Figure $4 \mathrm{~b}$ shows that for $d \geq 4 \mathrm{kpc}$ the hadronic $\gamma$-ray flux approaches the inverse Compton (IC) flux. The latter is almost insensitive to the source distance $d$. Therefore its value $\epsilon_{\gamma} F_{\gamma} \approx$ $10^{-2} \mathrm{eV} /\left(\mathrm{cm}^{2} \mathrm{~s}\right)$ at $10^{8}<\epsilon_{\gamma}<10^{13} \mathrm{eV}$ represents the lowest possible limit. The contribution of non-thermal bremsstrahlung (NB) emission is negligibly small.

\subsection{Simple IC emission model}

The closeness of the predicted hadronic and IC $\gamma$-ray fluxes might at first sight also be seen to justify the simple consideration that a situation is possible or even likely in which no nuclear particles are accelerated at all, but only electrons. The observed upper limit on the $\gamma$-ray flux might then be explained by the IC $\gamma$-ray emission in the cosmic microwave background (CMB) 
radiation field of the same accelerated electrons which also produce the observed synchrotron emission.

A simple estimate of the expected IC TeV-emission can be obtained from the relation $\epsilon_{\gamma} F_{\gamma}^{\mathrm{IC}}=v S_{\nu} w_{\mathrm{ph}} /\left(B_{\mathrm{d}}^{2} / 8 \pi\right)$ which is valid for the gamma ray energy $\epsilon_{\gamma}=1.5 \times$ $10^{10}(h v / \mathrm{eV})\left(10 \mu \mathrm{G} / B_{\mathrm{d}}\right) \mathrm{eV}$ assuming that IC scattering takes place in the Thompson regime. Here $w_{\mathrm{ph}} \approx 0.3 \mathrm{eV} / \mathrm{cm}^{3}$ is the CMB energy density. Since according to Fig. 2 we have $v S_{v} \approx 100 \mathrm{eV} /\left(\mathrm{cm}^{2} \mathrm{~s}\right)$ for $h v=1 \mathrm{keV}\left(v \approx 2 \times 10^{17} \mathrm{~Hz}\right)$, the expected IC $\gamma$-ray energy flux at $\epsilon_{\gamma}=15\left(10 \mu \mathrm{G} / B_{\mathrm{d}}\right) \mathrm{TeV}$ produced by the same electrons is $\epsilon_{\gamma} F_{\gamma}^{\mathrm{IC}} \approx 10\left(10 \mu G / B_{\mathrm{d}}\right)^{2} \mathrm{eV} /\left(\mathrm{cm}^{2} \mathrm{~s}\right)$. These relations show that the IC $\gamma$-ray flux exceeds the HEGRA upper limit unless the interior magnetic field is considerably smaller than $10 \mu \mathrm{G}$.

In order to illustrate the energy spectrum expected in the case of inefficient proton acceleration we present in Fig. $4 \mathrm{a}$ a IC $\gamma$-ray spectrum, calculated at $B_{\mathrm{d}}=20 \mu \mathrm{G}$ an with electron energy distribution function $f_{\mathrm{e}}(\epsilon)=A \epsilon^{-2} \exp \left(-\epsilon / \epsilon_{\max }\right)$, which corresponds to the test particle solution. The parameters $A$ and $\epsilon_{\max }=13 \mathrm{TeV}$ in this expression were determined by fitting the observed synchrotron flux $v S_{v}$ at $v=1 \mathrm{GHz}$ and $v=3 \times 10^{18} \mathrm{~Hz}$ (see Figs. 2 and 4).

As expected from the results of the nonlinear theory, this simple test particle theory which considers an inefficient production of nuclear CRs is quite inconsistent with the existing upper limit for the $\gamma$-ray production. Since the synchrotron emission is expected to come from those regions of the shock surface where the field is strongly amplified, the shock is a quasi-parallel shock. Therefore the ISM magnetic field which should have a strength of $5 \mu \mathrm{G}$ or less, should be MHD-compressed by a factor that is significantly less than the shock compression ratio. As a result an interior field strength $B_{\mathrm{d}}$ of about $10 \mu \mathrm{G}$ is adequate for this type Ia SN. In Fig. 4 a field strength $B_{\mathrm{d}}=20 \mu \mathrm{G}$ was chosen. It still overpredicts the $\gamma$-ray flux by an order of magnitude. Only for $B_{\mathrm{d}} \approx 70 \mu \mathrm{G}$ the predicted IC $\gamma$-ray flux would be as low as the observed HEGRA upper limit ${ }^{5}$. This result shows that the assumption of electron acceleration alone in SNRs has no physical basis and should be abandoned.

\subsection{Interior magnetic field strength from $X$-ray filaments}

We have also determined the interior magnetic field value from the observed linear thickness $L$ of X-ray filaments (see Völk et al. 2005a, for details). These values are given in Table 1 as $B_{\mathrm{d}}^{\prime}$ for different distances. Since the size $L$ increases proportional to $d$, the magnetic field strength $B_{\mathrm{d}}^{\prime} \propto L^{-2 / 3}$ (Berezhko $\&$ Völk 2004) decreases with increasing $d$. Therefore there is a growing discrepancy in the ratios between $B_{\mathrm{d}}$ and $B_{\mathrm{d}}^{\prime}$ : at $d=4.5 \mathrm{kpc} B_{\mathrm{d}}^{\prime} \approx 310 \mu \mathrm{G}$ which is already considerably smaller than $B_{\mathrm{d}} \approx 370 \mu \mathrm{G}$. $B_{\mathrm{d}}^{\prime} / B_{\mathrm{d}}$ is already considerably smaller than at $d=3.1 \mathrm{kpc}$. Therefore we might be able to constrain the source distance also from above, $d<4 \mathrm{kpc}$.

\section{Conclusions}

The last result led Völk et al. (2007) to the conclusion that one could constrain the source distance also from above, $d<4 \mathrm{kpc}$.

\footnotetext{
5 We remark that a similar result is found for SN1006, the other prototypical type Ia SN observed, where for $B_{\mathrm{d}} \approx 20 \mu \mathrm{G}$ the analogously calculated IC flux equals the HESS upper limit (Aharonian et al. 2005) for the $\gamma$-ray flux. Any smaller field $B_{\mathrm{d}}<20 \mu \mathrm{G}$ would overpredict the $\gamma$-ray flux.
}

However, this is not a strong argument, as the detailed data presented in Table 1 show. Therefore, regarding the expected $\gamma$-ray flux, we can only say the following: the increase in the kinetic energy of explosion to $E_{\mathrm{sn}}=1.2 \times 10^{51}$ erg requires a rather high source distance of $d>3.3 \mathrm{kpc}$ to explain the HEGRA nondetection. On the other hand, the rather low distance estimates from independent measurements summarized in the beginning of this section make it for this increased value of $E_{\mathrm{sn}}$ even more likely that the actual $\gamma$-ray flux from Tycho is "only slightly" below the HEGRA upper limit. The strong magnetic field amplification implies that the $\gamma$-ray flux is hadronically dominated, at least above photon energies of $1 \mathrm{GeV}$.

From the agreement between our theoretical solutions with the measurements of the discontinuity radii we conclude that there is new evidence for effective acceleration of nuclear particles in Tycho's SNR. A future $\gamma$-ray detection would therefore make the case for nuclear particle acceleration in SNRs incontrovertible.

Acknowledgements. H.J.V. is indebted to Wolfgang Hillebrandt for discussions about SN explosion energies and to Roger Chevalier for a discussion of the modification of the contact discontinuity by the Rayleigh-Taylor instability. E.G.B. and L.T.K. acknowledge the partial support by the Presidium of RAS (program No. 16) and by the Russian Foundation for Basic Research (grant 07-02-00221) and the hospitality of the Max-Planck-Institut für Kernphysik, where part of this work was carried out.

\section{References}

Aharonian, F. A., Akhperjanian, A., Barrio, J., et al. (HEGRA Collaboration) 2001, A\&A, 373, 292

Aharonian, F. A., Akhperjanian, A. G., Bazer-Bachi, A. R., et al. (HESS Collaboration) 2005, A\&A, 437, 135

Albinson, J. S., Tuffs, R. J., Swinbank, E., et al. 1986, MNRAS, 219, 427

Allen, G. E., Gotthelf, E. V., \& Petre, R. 1999, in 26th ICRC, Salt Lake City, 3, 480

Amato, E., \& Blasi, P. 2006, MNRAS, 371, 1251

Badenes, C., Borkowski, K. J., Hughes, J. P., et al. 2006, ApJ, 645, 1373

Ballet, J. 2006, Adv. Space Res., 37, 1902

Bamba, A., Yamazaki, R., Ueno, M., \& Koyama, K. 2003, ApJ, 589, 827

Bell, A. R. 1978, MNRAS, 182, 147

Bell, A. R. 2004, MNRAS, 353, 550

Bell, A. R., \& Lucek, S. G. 2001, MNRAS, 321, 433

Berezhko, E. G. 2005, Adv. Space Res., 35, 1031

Berezhko, E. G. 2008, Adv. Space Res., 41, 429

Berezhko, E. G., \& Ellison, D. C. 1999, ApJ, 526, 385

Berezhko, E. G., \& Krymsky, G. F. 1988, Soviet Phys.-Uspekhi, 12, 155

Berezhko, E. G., \& Völk, H. J. 1997, Astropart. Phys., 7, 183

Berezhko, E. G., \& Völk, H. J. 2004, A\&A, 419, L27

Berezhko, E. G., \& Völk, H. J. 2006, A\&A, 451, 981

Berezhko, E. G., Elshin, V. K., \& Ksenofontov, L. T. 1996, JETP, 82, 1

Berezhko, E. G., Ksenofontov, L. T., \& Völk, H. J. 2002, A\&A, 395, 943

Berezhko, E. G., Ksenofontov, L. T., \& Völk, H. J. 2003, A\&A, 412, L11

Blandford, R. D., \& Ostriker, J. P. 1978, ApJ, 221, L29

Blinnikov, S. I., Röpke, F. K., Sorokina, E. I., et al. 2006, A\&A, 453, 229

Buckley, J. H., Akerlof, C. W., Carter-Lewis, D. A., et al. 1998, A\&A, 329, 639 De Vaucouleurs, G. 1985, ApJ, 289, 5

Gamezo, V. N., Khokhlov, A. M., \& Oran, E. S. 2004, Phys. Rev. Lett., 92, 211102-1

Kirshner, R. P., Winkler, P. F., \& Chevalier, R. A. 1987, ApJ, 315, L135

Lagage, P. O., \& Cesarsky, C. J. 1983, ApJ, 125, 249

Long, K. S., Reynolds, S. P., Raymond, J. C., et al. 2003, ApJ, 586, 1162

Lucek, S.G., \& Bell, A. R. 2000, MNRAS, 314, 65

Marcowith, A., Lemoine, M., \& Pelletier, G. 2006, A\&A, 453, 193

McKenzie, J. F., \& Völk, H. J. 1982, A\&A, 116, 191

Niemiec, J., \& Pohl, M. 2007, in Proc. 30th ICRC, Merida;

[arXiv:0705.4459]

Nomoto, K., Thielemann, F.-K., \& Yokoi, K. 1984, ApJ, 268, 644

Parizot, E., Marcowith, A., Ballet, J., \& Gallant, Y. A. 2006, A\&A, 453, 387

Pelletier, G. Lemoine, M., \& Marcovith, A. 2006, A\&A, 453, 181

Prahl, J., \& Prosch, C. 1997, in Proc. 25th ICRC, Durban, 3, 217

Reynolds, S. P., \& Ellison, D. C. 1992, ApJ, 399, L75

Ptuskin, V. S., \& Zirakashvili, V. N. 2003, A\&A, 403, 1 
Ptuskin, V. S., \& Zirakashvili, V. N. 2005, A\&A, 429, 755

Ruiz-Lapuente, P. 2004, ApJ, 612, 357

Schwarz, U. J., Wakker, B. P., \& van Woerden, H. 1995, A\&A, 302, 364

Sinitsyna, V. G., Arsov, T. P., Malyshko, A. A., et al. 2007, in Proc. 30th ICRC, Merida

Smith, R. C., Kirshner, R. P., Blair, W. P., et al. 1991, ApJ, 357, 652

Tan, S. M., \& Gull, S. F. 1985, MNRAS, 216, 949

Vink, J., \& Laming, J. M. 2003, ApJ, 548, 758

Vladimirov, A., Ellison, D. C., \& Bykov, A. 2006, ApJ, 652, 1246

Völk, H. J. 2004, in Frontiers of Cosmic Ray Science, Proc. 28th ICRC, Tsukuba, 8, 29 ff, ed. T. Kajita, Y. Asaoka, A. Kawachi, Y. Matsubara, \& M. Sasaki (Tokyo, Japan, Universal Academy Press Inc.)

Völk, H. J., Berezhko, E. G., Ksenofontov, L. T., \& Rowell, G. P. 2002, A\&A 396,971
Völk, H. J., Berezhko, E. G., \& Ksenofontov, L. T. 2003, A\&A, 409, 563

Völk, H. J., Berezhko, E. G., \& Ksenofontov, L. T. 2005a, A\&A, 444, 893

Völk, H. J., Berezhko, E. G., \& Ksenofontov, L. T. 2005b, A\&A, 433, 229

Völk, H. J., Berezhko, E. G., \& Ksenofontov, L. T. 2007, Astrophys. Space Sci., 309,389

Völk, H. J., Berezhko, E. G., \& Ksenofontov, L. T. 2008, Adv. Space Res., 41, 473

Wang, C.-Y., \& Chevalier, R. A. 2001, ApJ, 549, 119

Warren, J. S., Hughes, J. P., Badenes, C., et al. 2005, ApJ, 634, 376

Zirakashvili, V. N., \& Ptuskin, V. S. 2008, ApJ, in press [arXiv:0801.4488v1]

Zirakashvili, V. N., Ptuskin, V. S., \& Völk, H. J. 2008, ApJ, in press [arXiv: 0801.4486v1] 